\title{
Myocardial blood flow imaging in patients with a left bundle branch block or ventricular- paced rhythm: "And therein, as the Bard would tell us, lies the rub"
}

\author{
Ibrahim Danad, $M D, P h D,{ }^{a}$ and Paul Knaapen, $M D, P h D^{a}$ \\ a Department of Cardiology, Amsterdam University Medical Center, Location Vu University \\ Medical Center, Amsterdam, The Netherlands
}

Received Feb 14, 2021; accepted Feb 15, 2021

doi: $10.1007 /$ s12350-021-02586-x

See related article, pp. 981 -988

\section{INTRODUCTION}

Nuclear-based imaging has been for more than a decade the cornerstone of noninvasive evaluation of myocardial perfusion in symptomatic patients. Ischemia assessment is of importance for guiding referral to invasive coronary angiography (ICA) and subsequent revascularization strategies. Although numerous trials such as the FAME, COURAGE and more recently the ISCHEMIA trial have indisputable demonstrated that stent placement shows no benefit in myocardial infarctions and cardiac death among stable coronary artery disease (CAD) patients, ${ }^{1-3}$ percutaneous coronary interventions (PCI) provide more and quicker symptom relief than optimal medical therapy. ${ }^{1,3,4}$ This therapeutic benefit of PCI may not be neglected and overlooked by clinicians by blindly staring at outcome data. Germane to this, it has to be said that the role of ischemia as a therapeutic target for prevention of future disease burden may still be a pivotal one. The recently published ISCHEMIA trial investigated the role of medical therapy versus revascularization in a large

Reprint requests: Ibrahim Danad, MD, PhD, Department of Cardiology, Amsterdam University Medical Center, Location VU University Medical Center, De Boelelaan 1117, 1081 HV Amsterdam, The Netherlands; i.danad@amsterdamumc.nl

J Nucl Cardiol 2021;28:989-91.

$1071-3581 / \$ 34.00$

Copyright (C) 2021 American Society of Nuclear Cardiology. cohort of patients with moderate to severe ischemia, a patient category neglected in previous studies. ${ }^{3}$ Despite this laudable achievement, paradoxically only $25 \%$ of included patients fulfilled the criterion of severe ischemia. Importantly, the definition of myocardial perfusionbased ischemia was mainly determined by the extent of ischemia and as such disregarding the depth of ischemia, which may be a potential important prognostic precursor for future cardiac events when treated conservatively. Therefore, functional imaging continues to have a pivotal role in selecting patients who likely will benefit from revascularization. However, diagnosis of ischemia in patients with a left bundle branch block (LBBB) or ventricular-paced rhythm (VPR) poses a diagnostic challenge raising the question which imaging technique is the most accurate for this task. And therein, as the Bard would tell us, lies the rub.

The present guidelines do not advocate one functional test over another in symptomatic patients with an intermediate pretest likelihood. ${ }^{5}$ The choice for a specific functional test is based on local availability and expertise. It is noteworthy to point out that a large body of evidence shows cardiac positron emission tomography (PET) to exhibit a higher diagnostic accuracy than conventional single-photon emission computed tomography (SPECT) myocardial perfusion imaging (MPI) for detection of CAD in stable chest pain patients. ${ }^{6}$ In earlier studies, in which ICA served as the reference standard, PET mainly excelled in reducing the number of false-positives due to its high spatial resolution and the use of a CT-based attenuation correction. An interesting and challenging group of patients are those with a LBBB or VPR. In these patients vasodilator stress imaging is advised. Of note, a LBBB may result from a sequela of ischemic heart disease, congenital heart 
disease, valvular heart disease, cardiomyopathies and can also be iatrogenic or degenerative in nature.

The presence of a LBBB or ventricular-paced rhythm clouds accurate judgment of myocardial perfusion images. Although, PET may perform better in stable CAD patients, the difference in diagnostic value between these two imaging modalities in patients with a LBBB or VPR is unknown. In this study of Vidula and colleagues, ${ }^{7}$ diagnostic performance of SPECT and (relative uptake) ${ }^{82} \mathrm{Rb}$ PET was compared using ICA as the reference standard. Germane to this, the number of included patients was only 55 of whom 17 underwent PET imaging. The lack of a functional reference standard leads to erroneous interpretations and in addition the small cohort of patients, especially in the PET group, does not permit any definite conclusions to be drawn. Furthermore it is noteworthy to point out that the authors did not exploit the full potential of cardiac PET using only visual grading and neglecting the potential additive value of quantification. Earlier studies have demonstrated that the perfusion defect seen in LBBB patients is due to relative hypoperfusion of the septal area. ${ }^{89}$ As such, absolute quantitative myocardial blood flow imaging may allow the discrimination of ischemia from relative hypoperfusion in these subset of patients. It may be postulated that a threshold below the ischemic cut-off should be used for the septal wall in the presence of a LBBB. Nevertheless, non-quantitative ${ }^{82} \mathrm{Rb}$ PET offered a better balance between sensitivity and specificity in comparison to SPECT in the present study. The results of the SPECT analysis are, to put it mildly, quite sobering. The low sensitivity of SPECT as a standalone tool is in keeping with the PACIFIC and Re-ASSESS trial. ${ }^{10,11}$ This is mainly attributable to its lower spatial resolution masking subtle endocardial perfusion abnormalities and the lack to quantify perfusion in absolute terms in combination with the unfavorable kinetics of the SPECT perfusion tracers. ${ }^{12}$ The use of coronary computed tomography angiography (CCTA) may overcome the limitations of functional testing in this category of patients with a LBBB or VPR that impedes accurate judgment of perfusion images. The fear that CCTA will produce similar results, namely a disproportionate high rate of false positive findings in LBBB patients is unjustified. A study by Clerc et al shows a similar prevalence of CAD in LBBB with a low-tointermediate pretest risk compared to controls with a similar cardiovascular risk profile. ${ }^{13}$ Furthermore, image quality was not hampered by the mechanical dyssynchronization in LBBB patients. Noteworthy, harnessing new advances in CT software, CCTA advanced from a purely anatomical modality to a tool that permits the functional assessment of epicardial lesions. In a metaanalysis, FFR-CT demonstrated high sensitivity (85-
93\%) and moderate specificity $(65-75 \%)$ when compared with invasive FFR. ${ }^{14}$ Interestingly, comprehensive measures of coronary atherosclerotic burden and adverse plaque characteristics using among others machine learning approaches were shown superior to MPI in detecting hemodynamic significant CAD in the recently published CREDENCE trial. ${ }^{15}$ These promising techniques allow the coupling of anatomical and functional measures by CCTA and as such reducing its rate of false positive findings.

In an editorial by Gupta et al in this Journal in 2019 on myocardial perfusion artifacts in $\mathrm{LBBB},{ }^{16}$ the following four research questions were raised that merited further investigation:

1. How is the diagnostic accuracy of MPI (PET and SPECT) in those with LBBB affected by the choice of pharmacological stress agent?

2. Does relative PET MPI have fewer artifacts in those with LBBB and improved diagnostic accuracy for CAD as compared to SPECT MPI?

3. Does the quantification of absolute MBF in interventricular septum and cardiac apex improve diagnostic accuracy?

4. If yes, what is the ideal PET radiotracer for quantifying MBF in patients with LBBB?

The effort of Dr Vidula and colleagues is laudable but incomplete when taken into consideration the abovementioned research questions. Based on the present study I would add the following questions for future research studies:

1. Does attenuation corrected SPECT MPI improve accuracy in LBBB patients?

2. Does the addition of calcium scoring of CCTA to SPECT MPI improve accuracy in LBBB and ventricular-paced rhythm patients?

3. Is CCTA a better choice for LBBB patients?

\section{Disclosures}

The authors declare no conflicts of interest.

\section{References}

1. Xaplanteris P, Fournier S, Pijls NHJ, Fearon WF, Barbato E, Tonino PAL, et al. Five-year outcomes with PCI guided by fractional flow reserve. N Engl J Med 2018;379:250-9.

2. Boden WE, O'Rourke RA, Teo KK, Hartigan PM, Maron DJ, Kostuk WJ, et al. Optimal medical therapy with or without PCI for stable coronary disease. N Engl J Med 2007;356:1503-16.

3. Maron DJ, Hochman JS, Reynolds HR, Bangalore S, O'Brien SM, Boden WE, et al. Initial invasive or conservative strategy for stable coronary disease. N Engl J Med 2020;382:1395-407. 
4. Al-Lamee R, Howard JP, Shun-Shin MJ, Thompson D, Dehbi H$\mathrm{M}$, Sen $\mathrm{S}$, et al. Fractional flow reserve and instantaneous wavefree ratio as predictors of the placebo-controlled response to percutaneous coronary intervention in stable single-vessel coronary artery disease. Circulation 2018;138:1780-92.

5. Knuuti J, Wijns W, Saraste A, et al. 2019 ESC guidelines for the diagnosis and management of chronic coronary syndromes. Eur Heart J 2020;41:407-77.

6. Mc Ardle BA, Dowsley TF, deKemp RA, Wells GA, Beanlands RS. Does rubidium-82 PET have superior accuracy to SPECT perfusion imaging for the diagnosis of obstructive coronary disease? A systematic review and meta-analysis. J Am College Cardiol 2012;60:1828-37.

7. Vidula MK, Wiener P, Selvaraj S, Khan MS, Salam UA, Rojulpote $\mathrm{C}$, et al. Diagnostic accuracy of SPECT and PET myocardial perfusion imaging in patients with left bundle branch block or ventricular-paced rhythm. J Nucl Cardiol 2020. https://doi.org/10. 1007/s12350-020-02398-5.

8. Vernooy K, Verbeek XA, Peschar M, Crijns HJ, Arts T, Cornelussen $\mathrm{RN}$, et al. Left bundle branch block induces ventricular remodelling and functional septal hypoperfusion. Eur Heart J 2005;26:91-8.

9. Knaapen P, van Campen LM, de Cock CC, Götte MJW, Visser CA, Lammertsma AA, et al. Effects of cardiac resynchronization therapy on myocardial perfusion reserve. Circulation 2004;110:646-51.

10. Danad I, Raijmakers PG, Driessen RS, Leipsic J, Raju R, Naoum C, et al. Comparison of coronary CT angiography, SPECT, PET, and hybrid imaging for diagnosis of ischemic heart disease determined by fractional flow reserve. JAMA Cardiol 2017;2:1100-7.
11. Sand NPR, Veien KT, Nielsen SS, Nørgaard BL, Larsen P, Johansen A, et al. Prospective comparison of FFR derived from coronary CT angiography with SPECT perfusion imaging in stable coronary artery disease: The ReASSESS study. JACC Cardiovasc Imaging 2018;11:1640-50.

12. Driessen RS, Raijmakers PG, Stuijfzand WJ, Knaapen P. Myocardial perfusion imaging with PET. Int J Cardiovasc Imaging 2017;33:1021-31.

13. Clerc OF, Possner M, Maire R, Liga R, Fuchs TA, Stehli J, et al. Association of left bundle branch block with obstructive coronary artery disease on coronary CT angiography: A case-control study. Eur Heart J Cardiovasc Imaging 2016;17:765-71.

14. Danad I, Szymonifka J, Twisk JWR, Norgaard BL, Zarins CK, Knaapen $P$, et al. Diagnostic performance of cardiac imaging methods to diagnose ischaemia-causing coronary artery disease when directly compared with fractional flow reserve as a reference standard: A meta-analysis. Eur Heart J 2017;38:991-8.

15. Stuijfzand WJ, van Rosendael AR, Lin FY, Chang H-J, van den Hoogen IJ, Gianni U, et al. Stress myocardial perfusion iImaging vs coronary computed tomographic angiography for diagnosis of invasive vessel specific coronary physiology: Predictive modeling results from the computed tomographic evaluation of atherosclerotic determinants of myocardial Ischemia (CREDENCE) trial. JAMA Cardiol 2020;5:1338-48.

16. Gupta K, Bajaj NS, Hage FG, Bhambhvani P. Myocardial perfusion artifacts in left bundle branch block: A diagnostic challenge. J Nucl Cardiol 2019. https://doi.org/10.1007/s12350-019-01717-9.

Publisher's Note Springer Nature remains neutral with regard to jurisdictional claims in published maps and institutional affiliations. 